\title{
Predistorsión Digital mediante Señales Enventanadas Tipo Chirp para la Linealización de Amplificadores de Potencia
}

\author{
L. Aladrén, P. García, J. de Mingo, P.L. Carro, C. Sánchez \\ Grupo de las Tecnologías de las Comunicaciones (GTC) \\ Instituto de Investigación en Ingeniería de Aragón (I3A) \\ Universidad de Zaragoza, Mariano Esquillor s/n, 50018, Zaragoza, Spain. \\ Tel. +34-976762707, Fax +34-976762043, e-mail: leticia@unizar.es
}

\begin{abstract}
Digital PreDistortion (DPD) is a well-known method to reduce nonlinear distortion in power amplifiers (PA). In a LTE uplink transmission, the modulation schemes are adaptive. Thus, the DPD function will have to be re-calibrated once the modulation changes. This fact increases the DPD computational cost and the required memory. If the DPD is not re-trained its linearity performance will decrease. This effect can be reduced using a suitable training signal. We propose several novel non-stationary calibration signals based on a windowed-chirp waveform. The envelope of these sequences is bounded by different windows used in signal processing such as Bartlett, Blackman, Hamming or Welch. Bartlett and Blackman windows generate calibration sequences with higher peak to average power ratio values than Welch. Welch envelope creates a signal with a more uniform probability density function (pdf) than the Hamming-window, whose pdf is similar to a Rayleigh distribution. The linearization strategy is based on capturing the described sequences at the PA input and output to extract the predistortion parameters. Once the predistorter functions are computed, we apply them on various LTE-transmissions and perform linearity measurements in terms of the adjacent channel leakage ratio to compare with the standard requirements. In all cases the maximum nonlinear distortion reduction is accomplished with the Chirp-Bartlett sequence (up to $37 \mathrm{dBc}$ when transmitting a QPSK-LTE-signal). Thus, good DPD performance is achieved when using the proposed Bartlett-Chirp in the DPD-training-stage. This avoids generating specific DPDs for each modulation scheme, saving computational cost, required memory, and increasing the system efficiency.
\end{abstract}

